\title{
32.3: Metal-Induced Unilaterally Crystallized Polycrystalline Silicon Thin- Film Transistor Technology for Active-Matrix Organic Light-Emitting Diode Displays with Reduced Susceptibility to Cross-Talk
}

\author{
Zhiguo Meng, Hoi S. Kwok and Man Wong- ${ }^{*}$ \\ Department of Electrical and Electronic Engineering \\ The Hong Kong University of Science and Technology. Clear Water Bay, Kowloon, Hong Kong
}

\begin{abstract}
In active-matrix organic light-emitting diode displays, a pixel in its non-emitting state is determined to be more sensitive to transistor leakage than one in its emitting state. An improved device design is proposed and implemented, employing gatemodulated lightly-doped drain to suppress transistor leakage and to improve image quality.
\end{abstract}

\section{Introduction}

Organic light-emitting diode (OLED) is challenging liquidcrystal (LC) as an alternative flat-panel display technology because of its ease of manufacturing due to its all solid-state nature and its relative merits of having a faster switching speed and being self-emitting with a wider viewing angle.

Unlike voltage-switched LC pixels, OLED pixels are currentdriven. Consequently, OLED-based active-matrix (AM) displays require thin-film transistors (TFTs) capable of delivering high drive current $\left(I_{\mathrm{on}}\right)$ in the on-state. While it is feasible to implement pixel transistors in AM-LC displays using n-channel amorphous silicon (a-Si) TFTs with relatively poor device characteristics, p-channel polycrystalline silicon (poly-Si) TFTs capable of driving significantly higher $I_{\text {on }}$ are required for implementing the pixel transistors in AM-OLED displays [1].

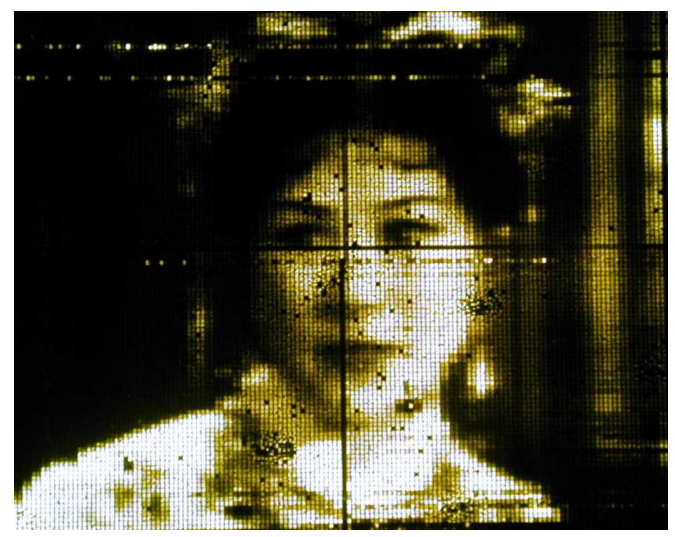

Figure 1. Typical image of a 160-by-120 AM-OLED display.

Metal-induced unilaterally crystallized (MIUC) poly-Si TFT technology [2] has been applied to implement AM-OLED displays (Fig. 1). While the brightness and uniformity of the two-transistor AM pixel (Fig. 2) is adequate, the display suffers from image quality degradation (Fig. 3) resulting from leakage current $\left(I_{\mathrm{lk}}\right)$ induced cross-talk [3]. It is presently shown that $I_{\mathrm{lk}}$, particularly its sensitivity to gate-induced drain leakage (GIDL) mechanism, is mainly responsible for the cross-talk.

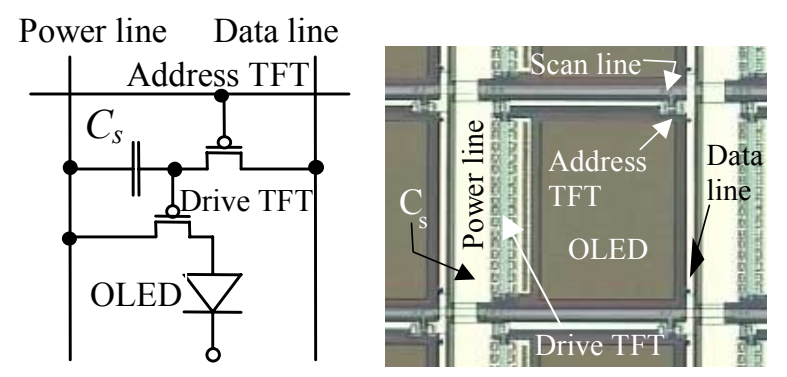

Figure 2. Circuit schematic (left) and photograph (right) of a two-transistor AM-OLED pixel.

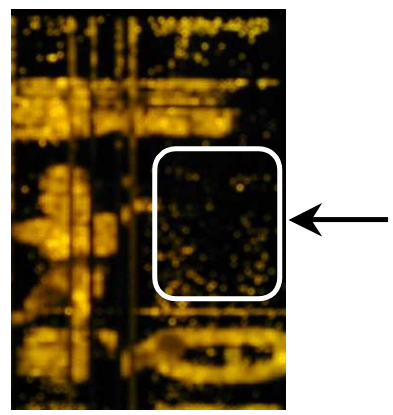

$I_{\mathrm{lk}}$ induced image degradation: nonemitting pixels unintentionally turned-on and become emitting.

Figure 3. Cross-talk induced image degradation in an AMOLED display.

GIDL can be reduced by employing lightly-doped drain (LDD) or offset drain structures [4,5]. However, TFTs incorporating conventional LDD with oxide spacers or offset drains suffer from higher parasitic source/drain resistance. This leads to reduced $I_{\mathrm{on}}$. An improved transistor design, in which the LDD is formed under poly-Si gate spacers, is presently proposed. The spacers are electrically connected to the gate. In the transistor "on" state, the LDD regions are accumulated and their resistance is lowered. This alleviates the negative impact of LDD resistance on $I_{\mathrm{on}}$. In the "off" state, the regions are depleted and the carrier concentration is lowered due to the gate-modulation, thus their effectiveness in reducing the magnitude of any penetrating drain electric field is potentially enhanced 


\section{Cross-Talk in OLED Pixels}

Cross-talk among OLED pixels can be illustrated using the two pixels shown in Figure 4. Pixel $P_{1}$ is not being actively addressed ( $V_{\text {scan, } 1}$ is high), while Pixel $\mathrm{P}_{2}$ is $\left(V_{\text {scan, } 2}\right.$ is low). The signal stored on $C_{\mathrm{s}}$ in $\mathrm{P}_{1}$ is $V_{\text {data, } 1}$ while that on the data line is $V_{\text {data, } 2}$, to be written into $\mathrm{P}_{2}$.

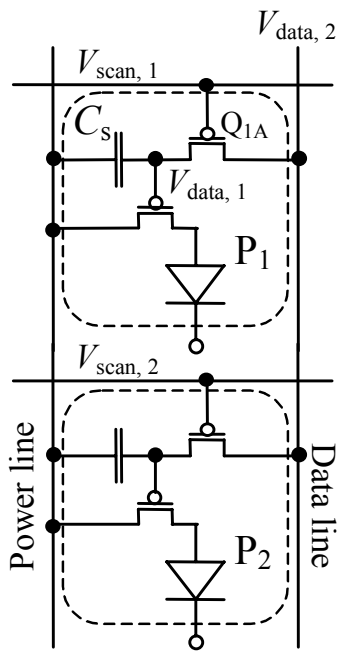

Figure 4. Circuit schematic illustrating leakage current induced cross-talk in an AM-OLED display.

Summarized in Table I are the possible cross-talk susceptible combinations of signal levels.

Table I. Cross-talk susceptible combinations of signal levels.

\begin{tabular}{c|c}
\hline Pixel $\mathrm{P}_{1}: V_{\text {scan }, 1}=$ high & Pixel $\mathrm{P}_{2}: V_{\text {scan }, 2}=$ low \\
\hline Non-emitting: $V_{\text {data, } 1}=$ high & Emitting: $V_{\text {data, } 2}=$ low \\
\hline Emitting: $V_{\text {data, } 1}=$ low & Non-emitting: $V_{\text {data, } 2}=$ high \\
\hline
\end{tabular}

With $\mathrm{P}_{1}$ non-emitting $\left(V_{\text {data, } 1}\right.$ high $)$ and $\mathrm{P}_{2}$ to be emitting $\left(V_{\text {data, } 2}\right.$ low), the $C_{\mathrm{s}}$ end of the p-channel address transistor $\left(\mathrm{Q}_{1 \mathrm{~A}}\right)$ acts as the source. Finite $I_{\mathrm{lk}}$ through $\mathrm{Q}_{1 \mathrm{~A}}$ causes $V_{\text {data, } 1}$ to drop, thus making the effective gate drive $\left(V_{\text {scan, } 1}-V_{\text {data, } 1}\right)$ on $\mathrm{Q}_{1 \mathrm{~A}}$ more positive. If GIDL were severe, $I_{\mathrm{lk}}$ would increase. Such "positive feedback" mechanism results in $V_{\text {data, } 1}$ being rapidly lowered. Consequently, a non-emitting pixel becomes emitting.

With $\mathrm{P}_{1}$ emitting ( $V_{\text {data, } 1}$ low) and $\mathrm{P}_{2}$ to be non-emitting $\left(V_{\text {data, } 2}\right.$ high), the "data line" end of $\mathrm{Q}_{1 \mathrm{~A}}$ acts as the source. Finite $I_{\mathrm{lk}}$ through $\mathrm{Q}_{1 \mathrm{~A}}$ causes $V_{\text {data, } 1}$ to increase. However $I_{\mathrm{lk}}$ is insensitive to GIDL because $V_{\text {data, } 2}$, hence the gate-drive on $\mathrm{Q}_{1 \mathrm{~A}}$, is fixed. Consequently, an emitting pixel is less susceptible to $I_{\mathrm{lk}}$-induced cross-talk than a non-emitting pixel.

\section{TFT Fabrication}

A summary of the fabrication process for the realization of the MIUC TFT with gate-modulated (gamo) LDD is shown in Figure 5. The process began with the deposition and patterning of a $30 \mathrm{~nm}$ thick a-Si active layer. $50 \mathrm{~nm}$ thick low-temperature oxide (LTO) as the gate dielectric, $300 \mathrm{~nm}$ a-Si as the gate electrode and $50 \mathrm{~nm}$ LTO as an etch-step were next deposited. Following the gate electrode patterning, $1 \times 10^{14} / \mathrm{cm}^{2}$ boron ions were implanted to fix the doping concentration for the LDD. A layer of 500nm thick a-Si was deposited and anisotropically etched to form the spacers.

A crystallization-inducing hole was opened in the gate dielectric on one end of the transistor island. Nickel was evaporated before heat treatment at $500^{\circ} \mathrm{C}$ for metal-induced lateral crystallization [6]. Any excess nickel was removed prior to heavy source/drain boron implantation, at a dose of $4 \times 10^{15} / \mathrm{cm}^{2}$. After dopant activation at $500^{\circ} \mathrm{C}$ for $3 \mathrm{hrs}, 500 \mathrm{~nm}$ LTO insulation layer was deposited. Contact holes were opened, $1 \mu \mathrm{m} \mathrm{Al}$ deposited and patterned, before the transistor was sintered in Forming gas at $420^{\circ} \mathrm{C}$ for $0.5 \mathrm{hr}$.

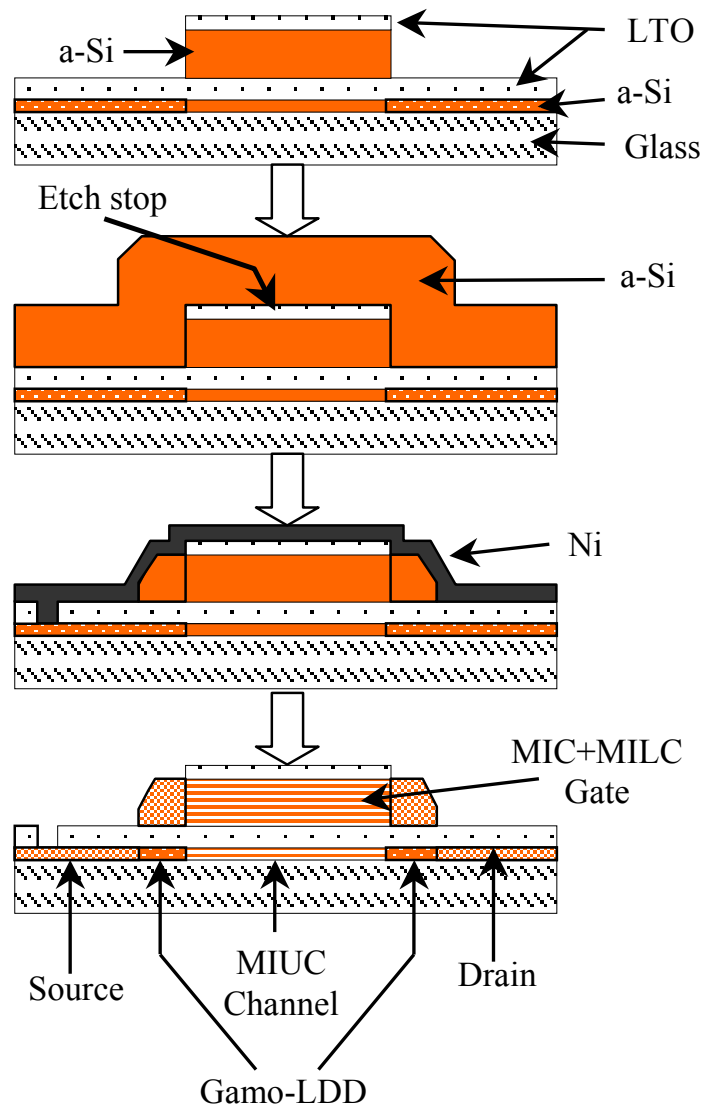

Figure 5. Schematic cross-sections showing the evolution of the structure of an MIUC TFT with gamo-LDD.

\section{Device Characteristics and Simulation}

The dependence of the drain current $\left(I_{\mathrm{d}}\right)$ on the gate voltage $\left(V_{\mathrm{g}}\right)$ of MIUC TFTs with and without gamo-LDD is shown in Figure 6. When $V_{\mathrm{g}}$ is positive, the devices are "off" in the accumulation state, hence $I_{\mathrm{d}}=I_{\mathrm{k}}$. For the device without gamo-LDD, $I_{\mathrm{lk}}$ increases exponentially with $V_{\mathrm{g}}$ - thus indicating the presence of a strong GIDL effect. The fact that $I_{\mathrm{lk}}$ is relatively independent of $V_{\mathrm{g}}$ in the off-state clearly indicates that GIDL is effectively suppressed in the TFT with gamo-LDD, while still preserving a relatively high $I_{\mathrm{on}}$.

A closer inspection of $I_{\mathrm{lk}}$ reveals that there are two valleys separated by a $V_{\mathrm{g}}$ difference of $\Delta V . I_{\mathrm{lk}}$ climbs out of the first valley as a result of an increase in drain electric field induced by 
a reduction in channel resistance due to accumulation. The second valley is reached when the carrier concentration, hence the resistance, of the LDD is gate-modulated to its most efficient drain field reduction state. Beyond the second valley, the LDD becomes inverted. The location of the maximum drain field shifts to the junction between the LDD and the heavily doped drain and $I_{\mathrm{lk}}$ again increases exponentially.

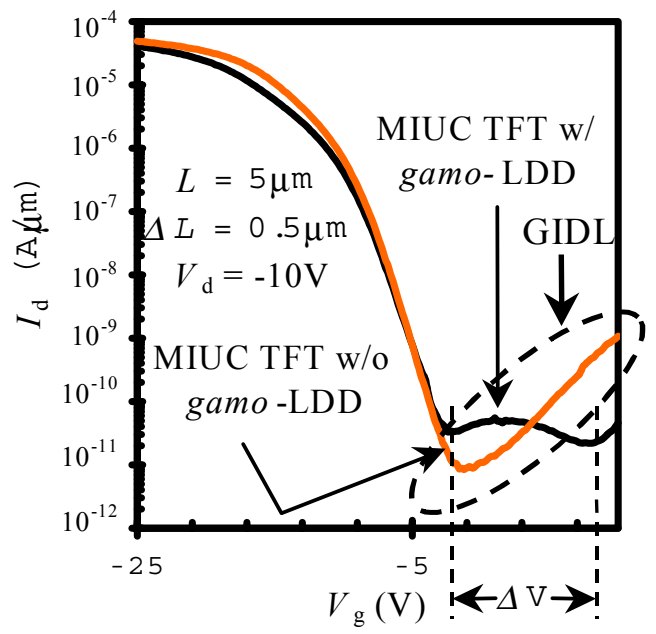

Figure 6. Transfer characteristics of MIUC TFT fabricated with and without gamo-LDD.

Shown in Figure 7 is the dependence of $I_{\mathrm{lk}}$ on $V_{\mathrm{d}}$ at a $V_{\mathrm{g}}$ of $10 \mathrm{~V}$ for a conventional bilaterally crystallized MILC TFT and MIUC TFT with or without gamo-LDD. For $I_{\mathrm{lk}}$ to reach a value of $10 \mathrm{pA} / \mu \mathrm{m}$, the approximate $V_{\mathrm{d}}$ values sustained by the three TFTs are $-2,-4,-10 \mathrm{~V}$, respectively. Gamo-LDD is clearly capable of significantly reducing GIDL. The dependence of $\Delta V$ on LDD implant dose is shown in Figure 8. While still preserving a small $I_{\mathrm{lk}}$, the largest (hence the most optimal) $\Delta V$ is obtained at a dose of $1 \sim 2 \times 10^{14} / \mathrm{cm}^{2}$.

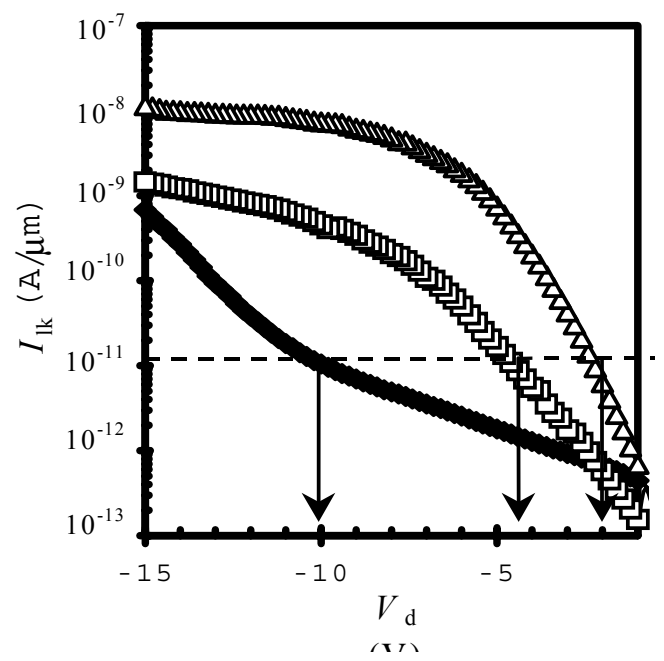

Figure 7. Dependence of $I_{\mathrm{lk}}$ on $V_{\mathrm{d}}$ of bilaterally crystallized MILC TFT (hollow triangle), MIUC TFT without (hollow square) and with gamo-LDD (filled diamond).
The dependence on $V_{\mathrm{g}}$ of the magnitude and location of the lateral electric field $\left(E_{\mathrm{x}}\right)$ in the drain/channel region for MIUC TFT without gamo-LDD and drain/LDD/channel region for MIUC TFT with gamo-LDD was studied using a device simulator and shown respectively in Figures 9 and 10. The dependence of the maximum $E_{\mathrm{x}}$ is summarized in Figure 11.

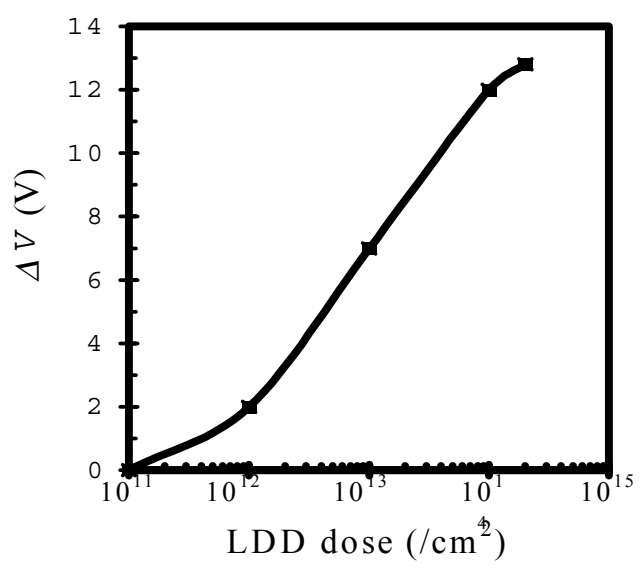

Figure 8. Dependence of $\Delta V$ on LDD implant dose.

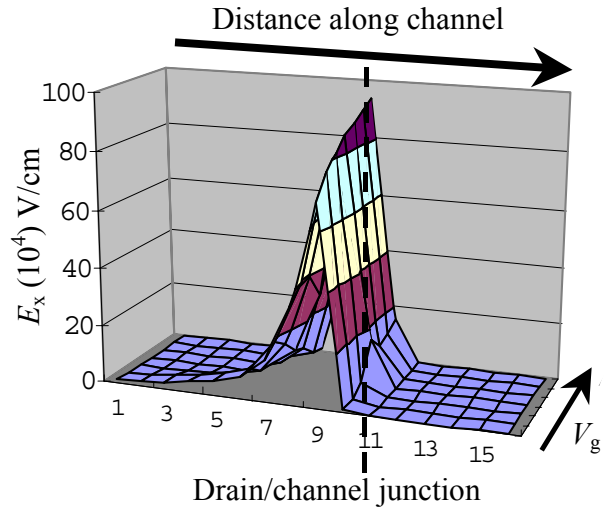

Figure 9. Dependence on $V_{\mathrm{g}}$ of the distribution of $E_{\mathrm{x}}$ in a conventional MIUC TFT without gamo-LDD.

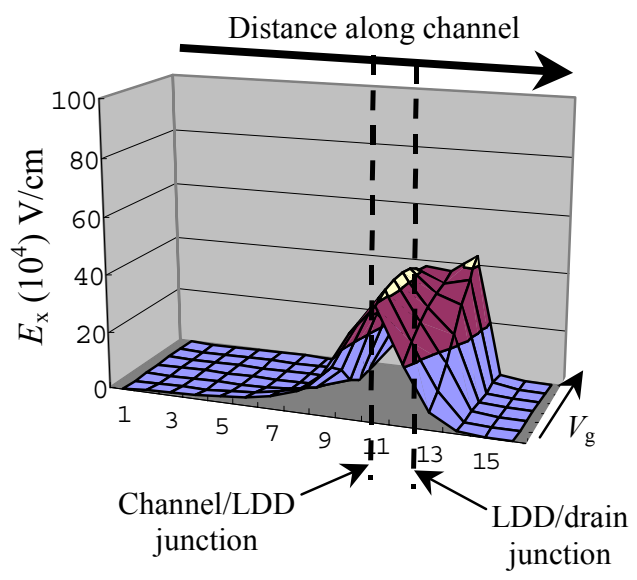

Figure 10. Dependence on $V_{\mathrm{g}}$ of the distribution of $E_{\mathrm{x}}$ in an MIUC TFT with gamo-LDD. 
At a $V_{\mathrm{d}}$ of $-12 \mathrm{~V}$, the maximum drain field $\left(E_{\mathrm{x}, \max }\right)$ increases from $\sim 0.6 \mathrm{MV} / \mathrm{cm}$ at $V_{\mathrm{g}}=0 \mathrm{~V}$ to $\sim 0.9 \mathrm{MV} / \mathrm{cm}$ at $V_{\mathrm{g}}=12 \mathrm{~V}$ (Fig. 9) for the TFT without gamo-LDD. Whereas for the TFT with gamo-LDD, the location of $E_{\mathrm{x}, \max }$ shifts from the channel/LDD junction to the $\mathrm{LDD} /$ drain junction as $V_{\mathrm{g}}$ is increased from $0 \mathrm{~V}$ to $12 \mathrm{~V}$ while its magnitude is relatively unchanged at $\sim 0.3$ to $\sim 0.4 \mathrm{MV} / \mathrm{cm}$.

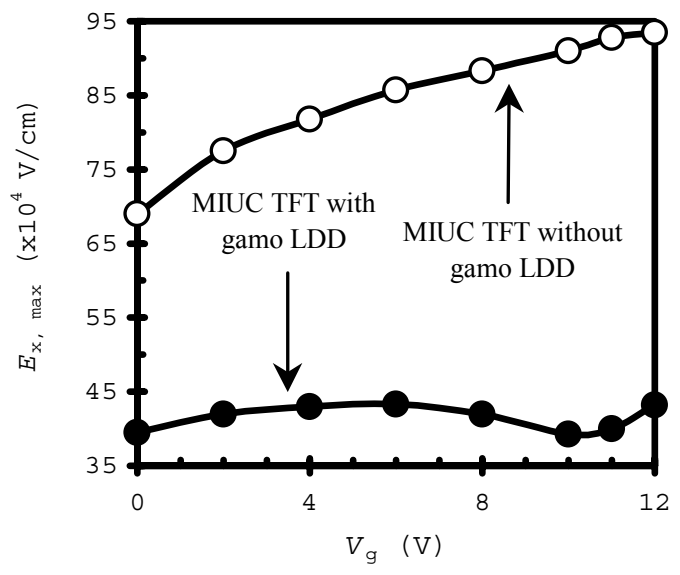

Figure 11. Dependence of $E_{\mathrm{x}, \max }$ on $V_{\mathrm{g}}$.

It can be seen in Figure 11 that for the TFT with gamo-LDD, not only is the reduction in $E_{\mathrm{x}, \max }$ (hence $I_{\mathrm{lk}}$ ) reproduced by the simulation but also its $V_{\mathrm{g}}$ dependence. The initial increase and subsequent decrease in the $E_{\mathrm{x}, \max }$ exactly mirror a similar dependence of $I_{\mathrm{lk}}$ on $V_{\mathrm{g}}$ shown in Figure 6.

The dependence of the maximum vertical electric field $\left(E_{\mathrm{y}, \max }\right)$ on $V_{\mathrm{g}}$ is shown in Figure 12. It is also reduced in a TFT with gamo-LDD.

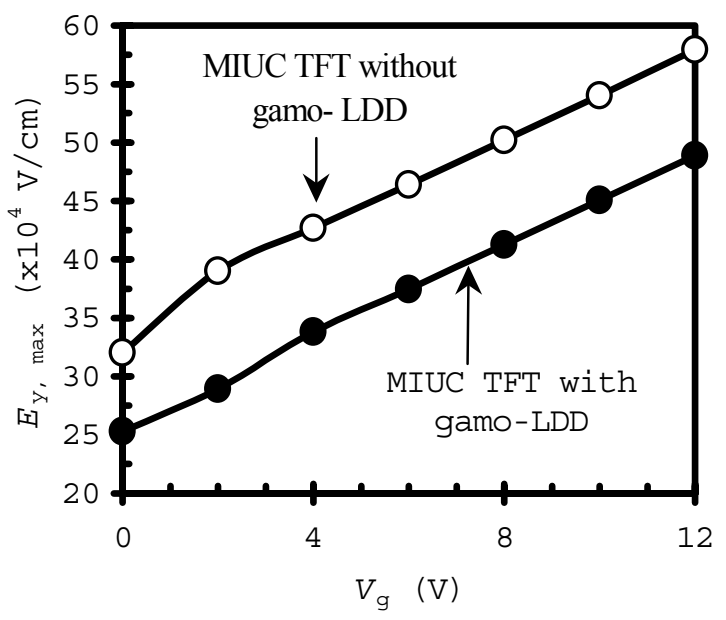

Figure 12. Dependence of $E_{\mathrm{y}, \max }$ on $V_{\mathrm{g}}$.

\section{Display Implementation}

As a demonstration, AM-OLED displays using TFTs with or without gamo-LDD have been fabricated. The images are compared in Figure 13. It can be seen that the display implemented using TFTs without the $I_{1 \mathrm{k}}$-reducing gamo-LDD suffers from severe cross-talk induced image degradation. A large number of non-emitting pixels are unintentionally turn on and become emitting. The display implemented using TFTs with gamo-LDD is clearly superior and does not suffer from similar $I_{\mathrm{lk}}$-induced image degradation.

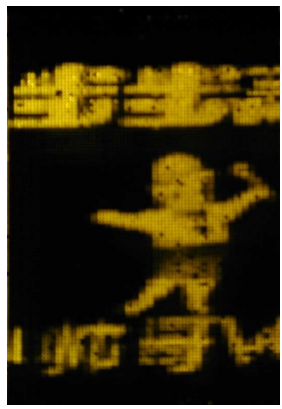

(a)

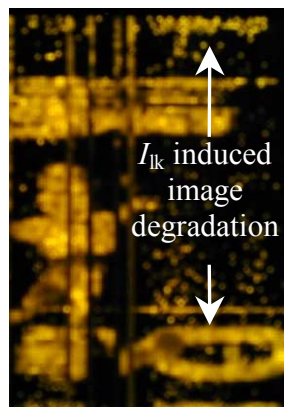

(b)
Figure 13. AM-OLED displays implemented using MIUC TFTs with (a) and without (b) gamo-LDD. $I_{\mathrm{lk}}$-induced image degradation is clearly visible in (b).

\section{Conclusion}

Without excessive sacrifice of the on-state drive current, leakage current in low-temperature metal-induced unilaterally crystallized polycrystalline silicon thin-film transistors has been reduced by incorporating a gate-modulated lightly-doped drain structure. Such structure reduces the strength of the electric field near the drain/channel junction region. The leakage current is typically less than $50 \mathrm{pA} / \mu \mathrm{m}$ at a $V_{\mathrm{d}}$ of $-10 \mathrm{~V}$ and a $V_{\mathrm{g}}$ of $10 \mathrm{~V}$. The new MIUC poly-Si TFTs are applicable to the realization of high quality AM-OLED displays and 3-dimensional integrated circuits.

\section{Acknowledgment}

This work was supported by a grant from the Research Grants Council of the Hong Kong Special Administrative Region.

\section{References}

[1] Meng, Z., Chen, H., Qiu, C., Kwok, H. S. and Wong, M. Digest of Technical Papers Vol. XXXII, Society for Information Display 2001 International Symposium. June/3-8/2001, San Jose, CA, USA. pp.380-383.

[2] Meng, Z., Wang, M. and Wong, M. IEEE Transactions on Electron Devices, Vol. 47(2). 2000. pp.404-409.

[3] Meng, Z., Ma, T. and Wong, M. Technical Digest, IEEE International Electron Device Meeting, Dec/3-5/2001, Washington D. C., USA. pp.755-758.

[4] Cao, Z. M., Plummer, J. D. and Saraswat, K. C. Technical Digest, IEEE International Electron Device Meeting. Dec/5-8/1993. Washington D.C., USA. pp.393-396.

[5] Takana, K., Arai, H. and Kohda, S. IEEE Electron Device Letters, Vol. 9(1). 1988. pp.23-25.

[6] Jin, Z., Bhat, G. A., Yeung, M., Kwok, H. S. and Wong, M. J. Appl. Phys., Vol 84(1). July/1998. pp.194-200. 